\title{
Plagiochila parvivittata Inoue var. siangensis var. nov. (Plagiochilaceae, Marchantiophyta) from Arunachal Pradesh, India
}

\author{
Siddhartha Singh Deo and D. K. Singh \\ Botanical Survey of India, Kolkata - 700064, India
}

Article history

Received: 6 January 2015

Accepted: 12 January 2016

Published online: 25 March 2016

(c) Singh Deo and Singh 2016

Publisher

Horizon e-Publishing Group

Corresponding Author

Dr. D.K. Singh

305D, Saraswati Apartment,

Gomti Nagar Extension Sector 4, Lucknow - 226 010, India

区 singh_drdk@rediffmail.com

\begin{abstract}
Plagiochila parvivittata Inoue var. siangensis var. nov. is described from West Siang district in Arunachal Pradesh, Eastern Himalaya, India. The new taxon differs from the typical variety in larger length/breadth ratio of leaves with fewer marginal teeth, (0-) 2-3 teeth along the dorsal margin of leaves near apex, terminal cell of marginal teeth 4-12 (-18) times longer than wide and a very distinct vitta area with the cells measuring $75-114 \times 15-21 \mu \mathrm{m}$ in size.
\end{abstract}

\section{Keywords}

Plagiochila parvivittata Inoue var. siangensis; Arunachal Pradesh; Eastern Himalaya; India; new variety

Singh Deo, S. and D. K. Singh 2016. Plagiochila parvivittata Inoue var. siangensis var. nov. (Plagiochilaceae, Marchantiophyta) from Arunachal Pradesh, India. Plant Science Today 3(1): 63-67. http://dx.doi.org/10.14719/pst.2016.3.1.185

\section{Introduction}

Grolle and So (1999) eastablished subsection Caulimammillosae Grolle \& M.L.So within the section Zonatae Carl, to include species of the genus Plagiochila with mammillose stem surface, having a distinct unistratose layer of bulging, thin-walled, hyaline cortical cells forming hyaloderm, and included Plagiochila aspericaulis Grolle \& M.L.So, $P$. caulimammillosa Grolle \& M.L.So, P. hyalodermica Grolle \& M.L.So and P. parvivittata Inoue in it. Recently, Singh and Singh (2011) recorded $P$. hyalodermica Grolle \& M.L.So in Indian bryoflora from Sikkim in the Eastern Himalaya, which formed the first report of the subsection in India.

During the course of ongoing studies on liverworts and hornworts of the Eastern Himalaya, the authors came across another interesting taxon belonging to this subsection quite similar to Plagiochila parvivittata - a species hitherto known only from Bhutan and Nepal, in its overall appearance. The Indian plants, however, differed consistently from $P$. parvivittata in larger length/breadth ratio of leaves with fewer marginal teeth, (0-) 2-3 teeth along the dorsal margin of leaves near apex, terminal cell of marginal teeth with larger length/breadth ratio and a very distinct vitta, and hence merit the status of a new taxon.

\section{Materials and Methods}

A critical morpho-taxonomic study on the Indian specimens of the genus Plagiochila collected from West Siang district of Arunachal Pradesh in Eastern Himalaya, which initially looked similar to $P$. parvivittata, and their comparison with the Type of the latter (D.G. Long 8443: Isotype) obtained on loan from Herbarium Haussknecht, Jena (JE) revealed the consistent differences in certain features of their leaves, and hence the taxonomic individuality of the two. Different morphological features were 
were studied and photographed under bright field biological research microscope (Nikon eclipse 50i) and Stereo zoom binocular microscope (Olympus SZ 51), and the illustrations were made using drawing attachment. The Holotype and the Isotype of the new taxon have been deposited in the Cryptogamic section of the Central National Herbarium of the Botanical Survey of India, Howrah (CAL).

\section{Taxonomic Description and Discussion}

Plagiochila parvivittata Inoue var. siangensis Singh Deo \& D.K.Singh, var. nov. (Figs. 1, 2)
Type: India, Eastern Himalaya, Arunachal Pradesh, West Siang district, Menchukha (between Zupuk and Damingla forests), $28^{\circ} 40^{\prime} \mathrm{N}, 94^{\circ} 03^{\prime} \mathrm{E}$, c. 3300 $\mathrm{m}$, 08.05.2011, S. Singh Deo, 50829A (Holotype and isotype: CAL).

Plants yellowish brown - dark brown in herbarium; shoots large, robust, 6-10 cm long, 3-5 $\mathrm{mm}$ wide including leaves, sparsely branched; branches lateral intercalary, restricted to the upper portion of the plants only. Stem elliptical in outline in transverse section, 400-590 × 320-430 $\mu \mathrm{m}, 16-28$ cells across diameter; cortical cells in $3-$ 4 layers; cells of the hyalodermis $13-29 \times 13-28$

Table 1. Comparative morphological account of different species of subsect. Caulimammillosae of the genus Plagiochila

\begin{tabular}{|c|c|c|c|c|c|}
\hline Characteristics & P. aspericaulis & P. caulimammillosa & P. hyalodermica & $\begin{array}{c}\text { P. parvivittata } \\
\text { var. parvivittata }\end{array}$ & $\begin{array}{l}\text { P. parvivittata var. } \\
\text { siangensis }\end{array}$ \\
\hline Shoot & $\begin{array}{l}\text { 1.0-1.2 cm long, } \\
1.5-1.6 \mathrm{~mm} \text { wide }\end{array}$ & $\begin{array}{l}\text { 5-6 cm long, } 2.8-3.2 \\
\text { mm wide }\end{array}$ & $\begin{array}{l}2-2.2 \mathrm{~cm} \text { long, } 3.5- \\
4.0 \mathrm{~mm} \text { wide }\end{array}$ & $\begin{array}{l}3.5-6 \mathrm{~cm} \text { long, } 3- \\
4.5 \mathrm{~cm} \text { wide }\end{array}$ & $\begin{array}{l}6-10 \mathrm{~cm} \text { long, } 3-5 \\
\text { mm wide }\end{array}$ \\
\hline Paraphyllia & $1-3$ celled & 1-3 celled & absent & absent & absent \\
\hline Stem & $\begin{array}{l}10-16 \text { cells across } \\
\text { in diameter, } \\
\text { cortical cells in } 3- \\
4 \text { layers }\end{array}$ & $\begin{array}{l}10-16 \text { cells across } \\
\text { diameter, cortical } \\
\text { cells in } 3-4 \text { layers }\end{array}$ & $\begin{array}{l}\text { 9-10 cells across } \\
\text { diameter, cortical } \\
\text { cells in } 3-4 \text { layers }\end{array}$ & $\begin{array}{l}\text { 18-32 cells across } \\
\text { diameter, cortical } \\
\text { cells in } 3-4 \text { layers }\end{array}$ & $\begin{array}{l}16-28 \text { cells across } \\
\text { diameter, cortical } \\
\text { cells in } 3-4 \text { layers }\end{array}$ \\
\hline $\begin{array}{l}\text { Hyalodermic } \\
\text { cells }\end{array}$ & $20-25 \times 24-30 \mu \mathrm{m}$ & $20-22 \times 20-24$ um & $20 \times 20-40 \mu \mathrm{m}$ & $\begin{array}{l}14-25(-31) \times \\
12.5-25 \mu \mathrm{m}\end{array}$ & $13-29 \times 13-28 \mu \mathrm{m}$ \\
\hline $\begin{array}{l}\text { Leaf shape } \\
\text { and size }\end{array}$ & $\begin{array}{l}\text { Broadly ovate to } \\
\text { triangular-ovate, } \\
\text { apex broadly } \\
\text { rounded, } 0.7-0.9 \\
\text { mm long, } 0.6-0.7 \\
\text { mm wide }\end{array}$ & $\begin{array}{l}\text { Broadly ovate to } \\
\text { triangular-ovate, } \\
\text { apex narrowly } \\
\text { rounded, } 2.0- \\
2.1 \mathrm{~mm} \text { long, } 1.3-1.5 \\
\text { mm wide }\end{array}$ & $\begin{array}{l}\text { Broadly ovate or } \\
\text { triangularly ovate, } \\
\text { apex subacute, } 1.9- \\
2.1 \mathrm{~mm} \text { long, } 1.1- \\
1.2 \mathrm{~mm} \text { wide }\end{array}$ & $\begin{array}{l}\text { Broadly ovate or } \\
\text { triangularly } \\
\text { ovate, apex } \\
\text { narrowly } \\
\text { rounded, } 2.8-3.2 \\
\text { mm long, } 2.5-3.1 \\
\text { mm wide }\end{array}$ & $\begin{array}{l}\text { Broadly ovate to } \\
\text { triangular-ovate, } \\
\text { apex narrowly } \\
\text { rounded, } 2.5-3.5 \\
\text { mm long, (1.1-) } 1.5- \\
2.6 \mathrm{~mm} \text { wide }\end{array}$ \\
\hline $\begin{array}{l}\text { Leaf length/ } \\
\text { width ratio }\end{array}$ & $1-1.15: 1$ & 1.1-1.3:1 & $1.6-1.7: 1$ & 0.9-1.1:1 & (1.2-) 1.4-1.8 (-2.2):1 \\
\hline $\begin{array}{l}\text { Dorsal margin } \\
\text { of leaf }\end{array}$ & $\begin{array}{l}\text { Entire, basal part } \\
\text { recurved, base } \\
\text { long decurrent }\end{array}$ & $\begin{array}{l}\text { Entire, strongly } \\
\text { revolute, recurved, } \\
\text { base long decurrent }\end{array}$ & $\begin{array}{l}7-8 \text { teeth along } \\
\text { distal part, slightly } \\
\text { recurved, base } \\
\text { long decurrent }\end{array}$ & $\begin{array}{l}8-13 \text { teeth } \\
\text { present } \\
\text { throughout or till } \\
\text { mid region, } \\
\text { recurved, base } \\
\text { long decurrent }\end{array}$ & $\begin{array}{l}2-3 \text { teeth near the } \\
\text { apical region or } \\
\text { entire, recurved, } \\
\text { base long decurrent }\end{array}$ \\
\hline $\begin{array}{l}\text { Ventral margin } \\
\text { of leaf }\end{array}$ & $\begin{array}{l}\text { Hardly decurrent, } \\
\text { slightly arched }\end{array}$ & $\begin{array}{l}\text { Hardly decurrent, } \\
\text { strongly arched }\end{array}$ & $\begin{array}{l}\text { Hardly decurrent, } \\
\text { slightly arched }\end{array}$ & $\begin{array}{l}\text { Moderately } \\
\text { decurrent, } \\
\text { strongly arched }\end{array}$ & $\begin{array}{l}\text { Moderately } \\
\text { decurrent, } \\
\text { strongly arched }\end{array}$ \\
\hline Marginal teeth & $\begin{array}{l}6-10 \text { per leaf, } 1-3 \\
\text { (-4) cells long, } 2-3 \\
\text { cells wide at base. }\end{array}$ & $\begin{array}{l}10-15 \text { per leaf, } 1-2 \\
\text { cells long, } 1-2 \text { cells } \\
\text { wide at base. }\end{array}$ & $\begin{array}{l}20-22 \text { per leaf, (1-) } \\
2-4 \text { cells long, (1-) } \\
2-3(-4) \text { cells wide } \\
\text { at base. }\end{array}$ & $\begin{array}{l}36-46 \text { per leaf, } 3- \\
10 \text { cells long, } 1-4 \\
\text { cells wide at } \\
\text { base. }\end{array}$ & $\begin{array}{l}\text { (7-) 15-27 (-30) per } \\
\text { leaf, (1-) 2-6 (-11) } \\
\text { cells long, (1-) 2-4 (- } \\
\text { 5) cells wide at base. }\end{array}$ \\
\hline $\begin{array}{l}\text { Terminal cell of } \\
\text { teeth }\end{array}$ & $\begin{array}{l}40 \times 12 \mu \mathrm{m}, \mathrm{l} / \mathrm{b} \\
\text { ratio approx. } 4: 1\end{array}$ & $\begin{array}{l}28 \times 16 \mu \mathrm{m}, \mathrm{l} / \mathrm{b} \text { ratio } \\
2-4: 1\end{array}$ & $\begin{array}{l}88 \times 20 \mu \mathrm{m}, 1 / \mathrm{b} \\
\text { ratio } 4-5: 1\end{array}$ & $\begin{array}{l}41-64 \times 8-12 \mu \mathrm{m} \\
1 / \mathrm{b} \text { ratio }(3-) 5-8: 1\end{array}$ & $\begin{array}{l}48-84 \times 4.5-12 \mu \mathrm{m}, \\
\text { l/b ratio } 4-12(-18): 1\end{array}$ \\
\hline Vitta cells & $\begin{array}{l}\text { Vitta area well } \\
\text { differentiated, } \\
\text { cells } 36-40(-44) \times \\
16-20 \mu \mathrm{m}\end{array}$ & $\begin{array}{l}\text { Vitta area well } \\
\text { differentiated, cells } \\
60-80(-100) \times 20-24 \\
(-30) \mu \mathrm{m}\end{array}$ & $\begin{array}{l}\text { Vitta area well } \\
\text { differentiated, cells } \\
60-80 \times 20 \mu \mathrm{m}\end{array}$ & $\begin{array}{l}\text { Vitta area } \\
\text { indistinct, cells } \\
51-85 \times 24-28 \\
\mu \mathrm{m}\end{array}$ & $\begin{array}{l}\text { Vitta area well } \\
\text { differentiated, cells } \\
75-114 \times 15-21 \mu \mathrm{m}\end{array}$ \\
\hline Perianth & $\begin{array}{l}\text { Perianth } \\
\text { campanulate }\end{array}$ & Perianth cylindrical & $\begin{array}{l}\text { Perianth not } \\
\text { known }\end{array}$ & $\begin{array}{l}\text { Perianth not } \\
\text { known }\end{array}$ & Perianth not known \\
\hline
\end{tabular}




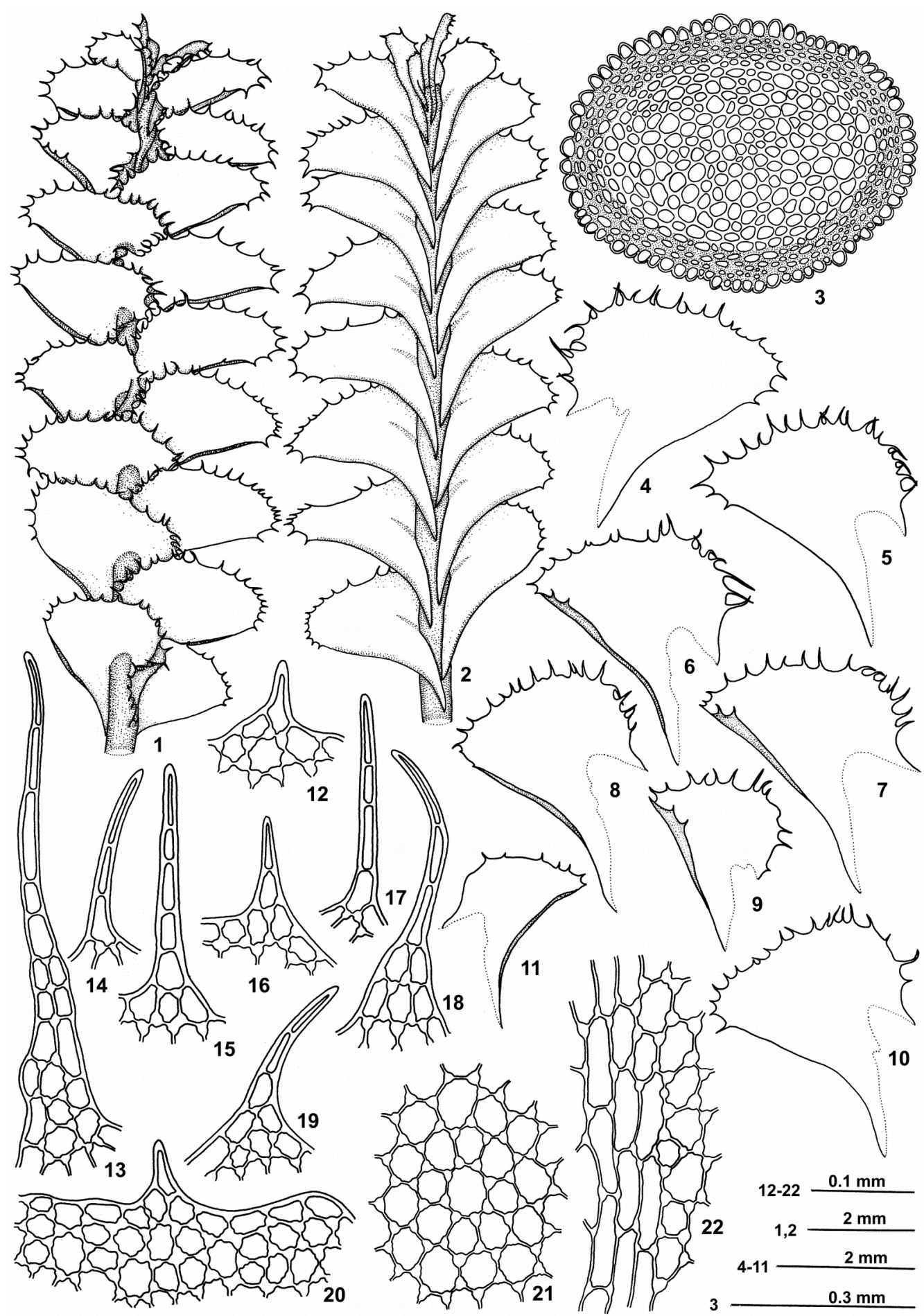

Fig. 1. Plagiochila parvivittata Inoue var. siangensis Singh Deo \& D.K.Singh (1) A portion of plant in ventral view. (2) A portion of plant in dorsal view. (3) Transverse section of the stem. (4-11) Leaves. (1219) Teeth along margin of leaf. (20) Marginal leaf cells. (21) Median leaf cells. (22) Basal leaf cells (showing vitta). (All figures drawn by S. Singh Deo from S. Singh Deo 50829A).

$\mu \mathrm{m}$, thin-walled, hyaline; inner cortical cells $5-10 \times$ 8-24 $\mu \mathrm{m}$, thick-walled, dark brown; medullary cells 18-43 × 14-42 $\mu \mathrm{m}$, thin-walled - moderately thick-walled, light brown - yellowish. Paraphyllia absent. Rhizoids few, restricted to basal portion of shoots. Leaves imbricate, succubous, obliquely inserted, broadly ovate - triangular-ovate, 2.5-3.5 $\mathrm{mm}$ long, (1.1-) 1.5-2.6 mm wide at middle, length/width ratio (1.2-) 1.4-1.8 (-2.2):1, dorsal margin more or less straight, recurved, entire or with 2-3 teeth near the apical region, base long decurrent, apex narrowly rounded, ventral margin strongly arched, dilated in basal region strongly dentate throughout, base moderately decurrent; marginal teeth spinose, (7-) 15-27 (-30) per leaf, (1-) 2-6 (-11) cells long, (1-) 2-4 (-5) cells wide at base, $2-5$ cells uniseriate towards apex; terminal cell long, triangular, acuminate, 48-84 × 4.5-12 $\mu \mathrm{m}$, length/breadth ratio 4-12 (-18):1; subterminal cells long, rectangulate, $36-60 \times 6-18 \mu \mathrm{m}$; marginal cells 


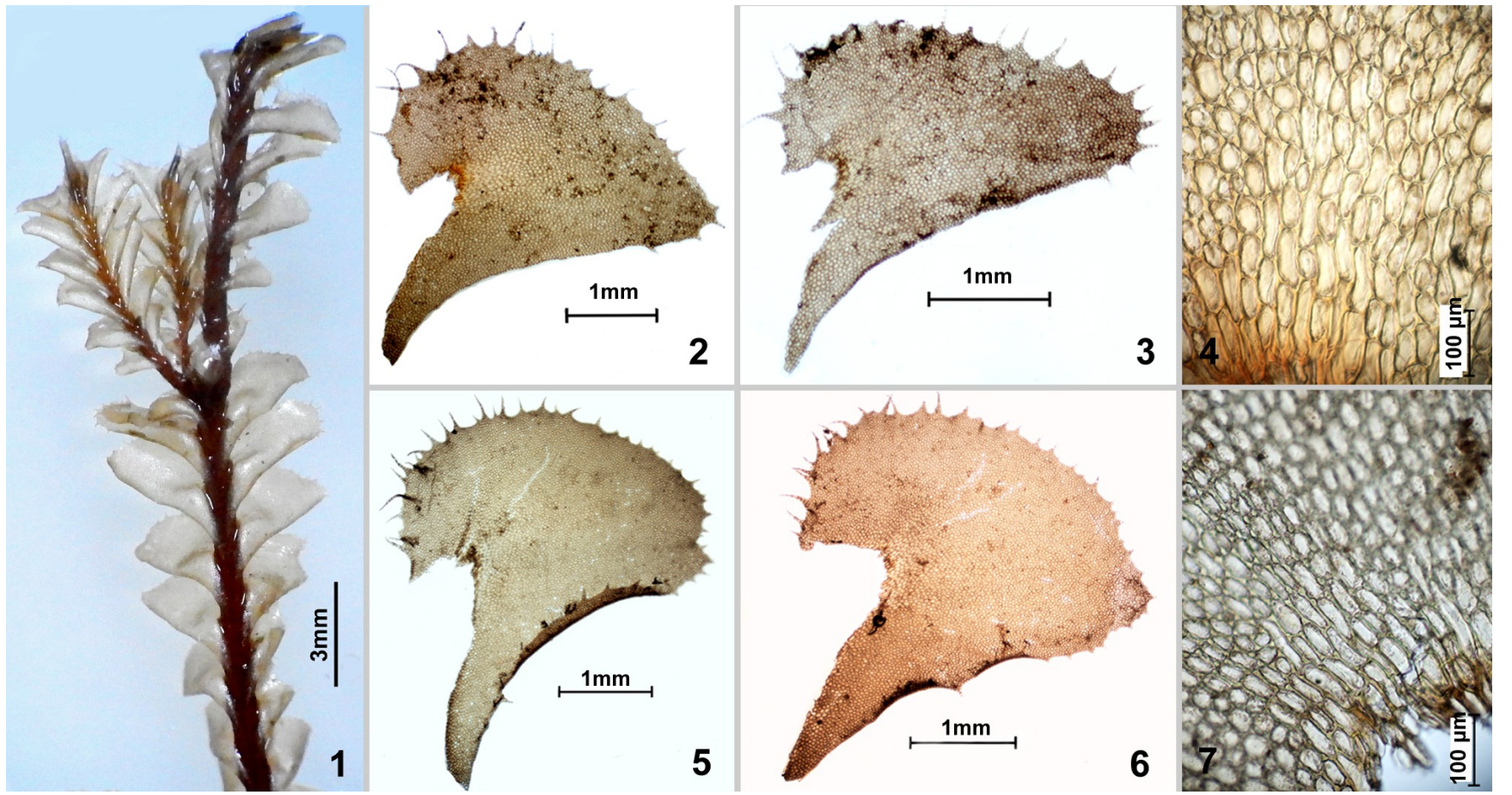

Fig. 2. Plagiochila parvivittata Inoue var. siangensis Singh Deo \& D.K.Singh (1) A portion of plant in dorsal view. (2,3) Leaves. (4) Basal leaf cells (showing vitta). Plagiochila parvivittata Inoue var. parvivittata $(5,6)$ Leaves. (7) Basal leaf cells (showing vitta) [Photomicrographs 1-4 from S. Singh Deo 50829A (CAL); 5-7 from D.G. Long 8443 (JE)].

near leaf apex quadrate -subquadrate, $12-24 \times 15-$ $30 \mu \mathrm{m}$, median leaf cells oval - suborbicular, 27-51 $\times 27-45 \mu \mathrm{m}$; vitta cells long, rectangulate, $75-114 \times$ 15-21 $\mu \mathrm{m}$, cells thin-walled, trigones nodulose, bulging, intermediate thickenings absent, or occasionally present in basal region; surface smooth. Underleaves absent. Androecia and gynoecia not seen.

Habitat: Lignicolous, on fallen logs in moist and shady conditions in temperate - subalpine forests mostly dominated by Rhododendron in association with Blepharostoma trichophyllum (L.) Dumort. and Hamatostrepta concinna Váňa \& D.G.Long.

Distribution: India [Eastern Himalaya (Arunachal Pradesh)], probably endemic.

Other specimens examined: Bhutan, Mongar District, Slope above Sengor, NW of Mongar, on mossy bank in moist Abies densa/Rhododendron forest, alt. 3500 m, D.G. Long 8443 (Isotype, JE).

Plagiochila parvivittata var. siangensis is characterized by 16-28 cells thick stem with mammillose surface, 3-4 layers of inner cortical cells (Fig. 1: 3); broadly ovate - triangular-ovate leaves with length/breadth ratio of (1.2-) 1.4-1.8 (2.2):1 and 7-30 marginal teeth (Figs. 1: 4-11; Figs. 2: 2,3); dorsal margin of leaves entire or with only 2-3 teeth near apex (Figs. 1: 4-11; Figs. 2: 2,3); teeth (1-) 2-6 (-11) cells long, (1-) 2-4 (-5) cells wide at base, 2-5 cells uniseriate towards apex (Figs. 1: 12-
20); terminal cells long, narrow, triangular, acuminate, $48-84 \times 4.5-12 \mu \mathrm{m}$, length/width ratio 4-12 (-18):1(Figs. 1: 12-20); leaf cells thin-walled with nodulose, bulging trigones (Figs. 1: 20-22; Fig. 2: 4) and a very distinct vitta area (Figs. 1: 22; Fig. 2: 4).

Subsection Caulimammillosae is comparatively new to the section Zonatae and is so far confined to Sino-Himalayan region (Bhutan, China, India and Nepal) only. Of the four species hitherto known under this subsection, $P$. aspericaulis is reported from China and Nepal, $P$. caulimammillosa is endemic to China, $P$. hyalodermica is known from China, Nepal and India, P. parvivittata from Bhutan and Nepal (Inoue, 1987; Grolle \& So, 1997, 1998, 1999; So, 2001; Pradhan \& Joshi, 2009; Singh \& Singh, 2011), whereas $P$. parvivittata var. siangensis is probably endemic to India.

$P$. parvivittata var. siangensis can be distinguished from the typical variety in leaves with nearly half the length/breadth ratio, 0.9-1.1:1 and profusely dentate margin with 36-46 marginal teeth, dorsal margin with 8-13 teeth present throughout or till mid region, terminal cell of marginal teeth (3-) 5-8 times longer than wide and indistinct vitta area with smaller vitta cells, 51-85 $\times 24-28 \mu \mathrm{m}$ in the latter (Table 1). The present taxon can be easily distinguished from other representatives of subsection Caulimammillosae on the basis of their comparative morphological accounts given in Table 1 . 


\section{Authors' contributions}

SSD worked out the specimens, carried out microscopic studies, prepared camera Lucida and photo-micrographic illustrations and draft description, and DKS coordinated the work, interpreted the results and confirmed the identity of the taxon after careful observation and evaluation of the morphological features of the new taxon as well as its allied species, drafted the discussion part, designed the submission and finalized the manuscript. Both the authors agreed to the final content of the manuscript.

\section{Acknowledgements}

The authors thank the Director, Botanical Survey of India for facilities and financial assistance to one of us (SSD) under 'Flora of India' project, Dr. H.-J. Zündorff, Curator, Herbarium Haussknecht, Jena (JE) for facilitating the study of Isotype of Plagiochila parvivittata, and the Chief Wildlife Warden, Arunachal Pradesh State Forest Department, for facilitating the exploration work.

\section{References}

Grolle, R. and M.L. So. 1997. Studies on Plagiochila in China I. Bryologist 100(4): 467-477. doi: 10.1639/00072745(1997)100[467:SOPICI]2.0.CO;2

Grolle, R. and M.L. So. 1998. Plagiochila caulimammillosa, a peculiar species from Yunnan, China. J. Bryol. 20: 41-49.

Grolle, R. and M.L. So. 1999. On the Plagiochila species of Sect. Zonatae with Paraphyllia or Mammillose Stems (Hepaticae). Syst. Bot. 24(3): 297-310. doi: $10.2307 / 2419690$

Inoue, H. 1987. Contributions to the knowledge of the Plagiochilaceae of southeastern Asia. XX. Studies on collections made by Dr. D. G. Long in Bhutan. Bull. Natl. Sci. Mus., Tokyo, B., Ser. B, Bot. 13(2): 41-51.

Pradhan, N. and S.D. Joshi. 2009. Liverworts and hornworts of Nepal: a synopsis. Botanica Orientalis - J. Pl. Sci. 6: 6975.

Singh, D. and D.K. Singh. 2011. Plagiochila hyalodermica Grolle \& M.L.So (Marchantiophyta: Plagiochilaceae) - an addition to indian bryoflora. Indian J. Forest. 34(4): 465-468.

So, M.L. 2001. Plagiochila (Hepaticae, Plagiochilaceae) in China. Syst. Bot. Monogr. 60: 1-214. doi: $10.2307 / 25027887$. 